\title{
UPPER AND LOWER BOUNDS FOR A REACTIVE-DIFFUSE SYSTEM WITH ARRHENIUS KINETICS
}

\author{
MOHAMMED AL-REFAI AND QUTAIBEH KATATBEH
}

Received 7 December 2005; Revised 31 May 2006; Accepted 5 June 2006

Comparison arguments are used to study a problem in combustion theory consisting of a nonlinear parabolic equation together with initial and boundary conditions. Upper and lower bounds for the problem are constructed. The lower solutions are used to determine whether the solution of the problem is increasing in time for certain initial condition. $\mathrm{Nu}$ merical results are presented for the slab, infinite cylinder, and unit sphere. The bounds are compared with the existing ones in the literature for the slab geometry.

Copyright (c) 2006 Hindawi Publishing Corporation. All rights reserved.

\section{Introduction}

In this paper we consider the nonlinear parabolic equation, which describes the reactivediffusive problem for a nonisothermal permeable catalyst pellet with first-order Arrhenius kinetics. The governing equation in the nondimensional form is

$$
\frac{\partial \theta}{\partial t}=\nabla^{2} \theta+\lambda^{2}(\beta-\theta) e^{\delta(\theta /(1+\theta))}, \quad \mathbf{x} \in \Omega, t>0,
$$

subject to homogeneous boundary condition of Dirichlet type and initial condition

$$
\theta(\mathbf{x}, 0)=r(\mathbf{x}) \geq 0 \text {. }
$$

Here $\Omega$ is a bounded domain of $\mathbb{R}^{N}$ and $\partial \Omega$ is the smooth enough boundary of $\Omega . \theta(\mathbf{x}, t)$ is the temperature of the reacting species, and $\beta, \delta$, and $\lambda$ are nonnegative parameters which represent the chemical heat release, the activation energy of the reaction, and the Thiele modulus, respectively. All variables are considered nondimensionalized. The full derivation of the system and extensive literature for early work can be found in [3]. The steadystate problem has been studied by many authors for the Dirichlet and Robin boundary conditions, see [5-9], and here is a summary of previous work. Kapila and Matkowsky [7] considered the problem on the slab and infinite cylinder and derived asymptotic expansion for the solution with large $\delta$. They found that the behavior of the solution is 
similar for both geometries and therefore only presented the results for the infinite cylinder. For the slab geometry the steady-state system has been reduced to a single equation by integrating the governing differential equation twice, see [5]. The literature shows that for certain values of $\delta$ and $\beta$ there exist $\lambda_{o}$ and $\lambda^{o}$ such that the steady-state system has multiple solutions for $\lambda_{o} \leq \lambda \leq \lambda^{o}$. Here $\lambda_{o}$ and $\lambda^{o}$ correspond to extinction and ignition limits, respectively, and the corresponding steady-state solutions are known as the middle solutions, whereas for $\lambda>\lambda_{o}$ and $\lambda<\lambda_{o}$ the unique steady-state solutions are known as the upper and lower solutions, respectively. The number of middle solutions depends on the geometry of the domain $\Omega$ and the boundary conditions [6-8]. Of interest are the values of $\lambda_{o}$ and $\lambda^{o}$. An attempt to evaluate these values was made in [7] for the slab and infinite cylinder geometries using asymptotic expansion approach. Recently, Al-Refai [1] has considered the problem with Dirichlet boundary conditions. He proved the existence of a nonnegative solution and derived sharp upper and lower bounds for the values of $\lambda$ and $\delta$ using comparison theory. Also in [2] he derived analytical upperand lower bounds for the extinction and ignition limits for the three geometries: slab, infinite cylinder, and unit sphere. Although the steady-state problem may have more than one solution, the problem with time-dependent has a unique solution provided that $0 \leq \theta(\mathbf{x}, 0) \leq \beta$ (see [10, page 42]).

In this paper, we study the time-dependent problem in the slab $[0,1]$, in the unit sphere, and infinite cylinder. In Section 2, we write some preliminary results for the system which will be used through the text. In Section 3, we construct upper and lower solutions for the problem (1.1)-(1.2). In Section 4, we present some numerical results in the three geometries. Finally, we write some concluding remarks in Section 5.

\section{A preliminary result}

We have the problem

$$
\begin{gathered}
P \theta=\frac{\partial \theta}{\partial t}-\nabla^{2} \theta-\lambda^{2} g(\theta)=0, \quad \mathbf{x} \in \Omega, t>0, \\
\theta(\mathbf{x}, t)=0, \quad \mathbf{x} \in \partial \Omega \\
\theta(\mathbf{x}, 0)=r(\mathbf{x}) \geq 0
\end{gathered}
$$

where $g(\theta)=(\beta-\theta) e^{\delta(\theta /(1+\theta))}$. A well known result for the system is that $0 \leq \theta(\mathbf{x}, t) \leq \beta$ provided that $r(x) \leq \beta$. If $\delta \beta \leq 1$, then

$$
g^{\prime}(\theta)=-\frac{e^{\delta(\theta /(\theta+1))}}{(\theta+1)^{2}}\left[\theta^{2}+(2+\delta) \theta+1-\delta \beta\right]<0,
$$

and the corresponding steady-state problem has a unique solution, see $[2,10]$. While, for $\delta>4+4 / \beta$, the steady-state problem may have more than one solution. The following result will be used in this paper. 
Proposition 2.1. Consider the problem in (2.1) with $g^{\prime}(\theta)$ being bounded.

(i) If $(\partial \theta / \partial t)(\mathbf{x}, 0)<0$, then $(\partial \theta / \partial t)(\mathbf{x}, t)<0$ for all $\mathbf{x} \in \Omega$, and $t \geq 0$.

(ii) If $(\partial \theta / \partial t)(\mathbf{x}, 0)>0$, then $(\partial \theta / \partial t)(\mathbf{x}, t)>0$ for all $\mathbf{x} \in \Omega$, and $t \geq 0$.

For the proof one can see $[4,12]$.

\section{Upper and lower bounds}

To construct upper and lower bounds for the problem we use maximum principle for parabolic equations, see [11, page 187]. Let $w(\mathbf{x}, t)$ and $u(\mathbf{x}, t)$ satisfy

$$
\begin{gathered}
P w \leq 0 \leq P u, \quad \mathbf{x} \in \Omega, t>0, \\
w(\mathbf{x}, t) \leq 0 \leq u(\mathbf{x}, t), \quad \mathbf{x} \in \partial \Omega, \\
w(\mathbf{x}, 0) \leq r(\mathbf{x}) \leq u(\mathbf{x}, 0) .
\end{gathered}
$$

Then $w(\mathbf{x}, t)$ and $u(\mathbf{x}, t)$ are lower and upper solutions for the problem in (2.1), respectively, $w(\mathbf{x}, t) \leq \theta(\mathbf{x}, t) \leq u(\mathbf{x}, t)$, as long as both exist.

Let $\lambda_{1}$ be the first eigenvalue and $\phi_{1}$ the corresponding normalized, with respect to $L^{2}$-norm, eigenfunction of

$$
\begin{gathered}
\nabla^{2} \phi=-\lambda \phi, \quad \mathbf{x} \in \Omega, \\
\phi=0, \quad \mathbf{x} \in \partial \Omega .
\end{gathered}
$$

It is easily obtained that $\phi_{1}=\sqrt{2} \sin (\pi x),(1 / \sqrt{2 \pi})(\sin (\pi x) / x)$, and $J_{0}\left(\gamma_{0} x\right)$, for the slab, spherical, and cylindrical geometries, respectively. Here $J_{0}\left(\gamma_{0} x\right)$ is the Bessel function of order zero, $\gamma_{0}=2.404825 \ldots$ is the first zero of $J_{0}(x)$, and $0 \leq x \leq 1$. In all cases, the first eigenfunction $\phi_{1}$ is nonnegative in $\Omega$.

3.1. Bounds when $g(\theta)$ is decreasing. We derive upper and lower solutions for the problem when $\delta \beta \leq 1$ and so $g(\theta)$ is decreasing. The function $g(\theta)$ has only one inflection point $\theta^{0}=(\delta \beta-2 \beta-2) /(\delta+2+2 \beta)$, and $g(\theta)$ is concave up for $\theta<\theta^{0}$ and concave down for $\theta>\theta^{0}$. For $\delta \beta \leq 1$, we have $\theta^{0}<0$, and therefore, $g(\theta)$ is concave down on $[0, \beta]$.

Theorem 3.1. Let $\phi_{1}$ and $\lambda_{1}$ be as defined in (3.2) and let $\phi_{1 m}$ be the maximum of $\phi_{1}$ on $\Omega$. Let $k(t)$ be the solution of the IVP

$$
\begin{gathered}
k^{\prime}(t)=\lambda^{2} g(k(t))-\lambda_{1} k(t), \\
k(0)=k_{0} .
\end{gathered}
$$

Then $k_{0} \leq k(t) \leq k_{m}$ and $w(\mathbf{x}, t)=k(t)\left(\phi_{1}(\mathbf{x}) / \phi_{1 m}\right)$ is a lower solution of (2.1). Here $k_{m}$ is the unique solution of $g\left(k_{m}\right)=\left(\lambda_{1} / \lambda^{2}\right) k_{m}$ and $k_{0}=k(0)$ is chosen such that $k_{0}\left(\phi_{1}(\mathbf{x}) / \phi_{1 m}\right) \leq$ $r(\mathbf{x})$.

Proof. Since $g(0)=\beta>0$, we have $\left(\lambda_{1} / \lambda^{2}\right) u \leq g(u)$ for $0 \leq u \leq k_{m}$ and $\left(\lambda_{1} / \lambda^{2}\right) u \geq g(u)$ for $k_{m} \leq u \leq \beta$. If $k_{0}<k_{m}$, then $k^{\prime}(t) \geq 0$ and $k(t)$ is increasing with equilibrium value $k_{m}$, 
4 Bounds for a reactive-diffuse system

and therefore, $k_{0} \leq k(t) \leq k_{m}$. The analogous result is obtained if $k_{m} \leq k_{0} \leq \beta$, but $k(t)$ is decreasing. Now,

$$
\begin{aligned}
P w & =k^{\prime} \frac{\phi_{1}}{\phi_{1 m}}+\lambda_{1} k \frac{\phi_{1}}{\phi_{1 m}}-\lambda^{2} g\left(k \frac{\phi_{1}}{\phi_{1 m}}\right) \\
& =\frac{\phi_{1}}{\phi_{1 m}} \lambda^{2} g(k)-\lambda^{2} g\left(k \frac{\phi_{1}}{\phi_{1 m}}\right) \leq \lambda^{2}\left[g(k)-g\left(k \frac{\phi_{1}}{\phi_{1 m}}\right)\right] .
\end{aligned}
$$

Since $k(t)\left(\phi_{1} / \phi_{1 m}\right) \leq k(t)$ and $g$ is decreasing, we have $P w \leq 0$, which together with $w(\mathbf{x}, 0) \leq r(\mathbf{x})$ proves that $w$ is a lower solution of $(2.1)$.

THEOREM 3.2. Let $\psi$ be the solution of

$$
\begin{gathered}
\nabla^{2} \psi=-1, \quad \mathbf{x} \in \Omega, \\
\psi=0, \quad \mathbf{x} \in \partial \Omega .
\end{gathered}
$$

Then $\psi \geq 0$, and $u(\mathbf{x}, t)=h(t) \psi(x)$ is an upper solution of (2.1), where

$$
h(t)=h_{0}-\frac{1}{\lambda^{2} g^{\prime}(0)}\left[1-e^{\lambda^{2} g^{\prime}(0) t}\right],
$$

and $h_{0}=h(0)$ is chosen such that $h_{0} \geq \lambda^{2} \beta$ and $h_{0} \psi(\mathbf{x}) \geq r(\mathbf{x})$.

Proof. To show that $\psi \geq 0$, let $\xi=-\psi$, then $\xi$ satisfies $\nabla^{2} \xi=1 \geq 0$, and $\xi=0$ on $\partial \Omega$. Using maximum principle of elliptic equations (see [11, page 64]), we have $\xi \leq 0$, and hence $\psi \geq 0$. Since $g^{\prime}(0)=\delta \beta-1 \leq 0$, it is not difficult to see that $h(t)$ is increasing with

$$
h_{0} \leq h(t) \leq h_{0}+\frac{1}{\lambda^{2}(1-\delta \beta)},
$$

and it is the unique solution of the IVP

$$
\begin{gathered}
h^{\prime \prime}-\lambda^{2} g^{\prime}(0) h^{\prime}=0, \\
h(0)=h_{0}>0, \quad h^{\prime}(0)=1 .
\end{gathered}
$$

Now, $P u=h^{\prime} \psi+h-\lambda^{2} g(h \psi)$ and

$$
\frac{\partial P u}{\partial t}=h^{\prime \prime} \psi+h^{\prime}-\lambda^{2} h^{\prime} \psi g^{\prime}(h \psi)=\left(h^{\prime \prime}-\lambda^{2} h^{\prime} g^{\prime}(h \psi)\right) \psi+h^{\prime} .
$$

Since $h(t) \psi \geq 0$ and $g^{\prime}$ is decreasing, we have

$$
\frac{\partial P u}{\partial t} \geq\left(h^{\prime \prime}-\lambda^{2} h^{\prime} g^{\prime}(0)\right) \psi+h^{\prime}(t)=h^{\prime}(t) .
$$

Integrate the above inequality from 0 to $t$ to get

$$
P u-P u(0) \geq h(t)-h(0),
$$


or

$$
P u \geq \psi+h(t)-\lambda^{2} g\left(h_{0} \psi\right) \geq h(t)-\lambda^{2} \beta \geq 0
$$

which together with $u(\mathbf{x}, 0)=h_{0} \psi(\mathbf{x}) \geq r(\mathbf{x}) \geq 0$ proves that $u$ is an upper solution of (2.1).

3.2. Lower solutions for $\delta>4+4 / \beta$. When $\delta>4+4 / \beta$, the inflection point $\theta^{0}=$ $(\beta(\delta-2)-2) /(\delta+2+2 \beta) \in[0, \beta]$. Let $\theta^{*} \in[0, \beta]$ be the smallest solution of $\left(\lambda_{1} / \lambda^{2}\right) \theta=$ $g(\theta)$ and $\lambda_{1} / \lambda^{2}=g^{\prime}(\theta)$, and let $\lambda^{*}$ be the corresponding value of $\lambda$, see Figure 3.1. For the exact values of $\theta^{*}$ and $\lambda^{*}$, one is referred to [2]. We have the following.

Proposition 3.3. (1) $\theta^{0}=(\beta(\delta-2)-2) /(\delta+2 \beta+2)>\theta^{*}=(\beta(\delta-2)-\sqrt{\beta \delta(\beta \delta-4 \beta-4)}) /$ $2(\beta+\delta)$ for $\delta>4+4 / \beta$.

(2) The function $h(\theta)=g(\theta)-\left(\lambda_{1} / \lambda^{2}\right) \theta$ is decreasing in $\left[0, \theta^{*}\right]$ for $\lambda \leq \lambda^{*}$.

Proof. (1) It is enough to show that

$$
\frac{\beta(\delta-2)-2}{\delta+2+2 \beta}>\frac{\beta(\delta-2)}{2(\beta+\delta)},
$$

or

$$
2(\beta+\delta)[\beta(\delta-2)-2]>\beta(\delta-2)(\delta+2 \beta+2)
$$

The last inequality is equivalent to

$$
\beta \delta^{2}-\delta(4 \beta+4) \geq 0
$$

Now, $4 \beta+4<\beta \delta$ and hence $-\delta(4 \beta+4)>-\beta \delta^{2}$, which proves (3.15).

(2) Since $\theta^{*}<\theta^{0}$, we have $g^{\prime}(\theta)$ increasing in $\left[0, \theta^{*}\right]$ and hence $h^{\prime}(\theta)=g^{\prime}(\theta)-\lambda_{1} / \lambda^{2} \leq$ $g^{\prime}\left(\theta^{*}\right)-\lambda_{1} /\left(\lambda^{*}\right)^{2}=0$, which proves the result.

Theorem 3.4. Let $\phi_{1}$ and $\lambda_{1}$ be as defined in (3.2) and let $\phi_{1 m}$ be the maximum of $\phi_{1}$ on $\Omega$. For $\lambda \leq \lambda^{*}$, let $k(t)$ be the solution of the IVP

$$
\begin{gathered}
k^{\prime}(t)=\frac{1}{\phi_{1 m}}\left\{\lambda^{2} g\left(k(t) \phi_{1 m}\right)-\lambda_{1} k(t) \phi_{1 m}\right\}, \\
k(0)=k_{0},
\end{gathered}
$$

where $k_{0} \leq k_{M}$ is chosen such that $k_{0} \phi_{1}(\mathbf{x}) \leq r(\mathbf{x})$, and $k_{M}$ is the solution (the smallest solution if there is more than one) of $\lambda^{2} g\left(k_{M} \phi_{1 m}\right)=\lambda_{1} k_{M} \phi_{1 m}$, see Figure 3.1. Then $k(t)$ is an increasing function, with $k_{0} \leq k(t) \leq k_{M}$, and $w(\mathbf{x}, t)=k(t) \phi_{1}(\mathbf{x})$ is a lower solution of (2.1). 


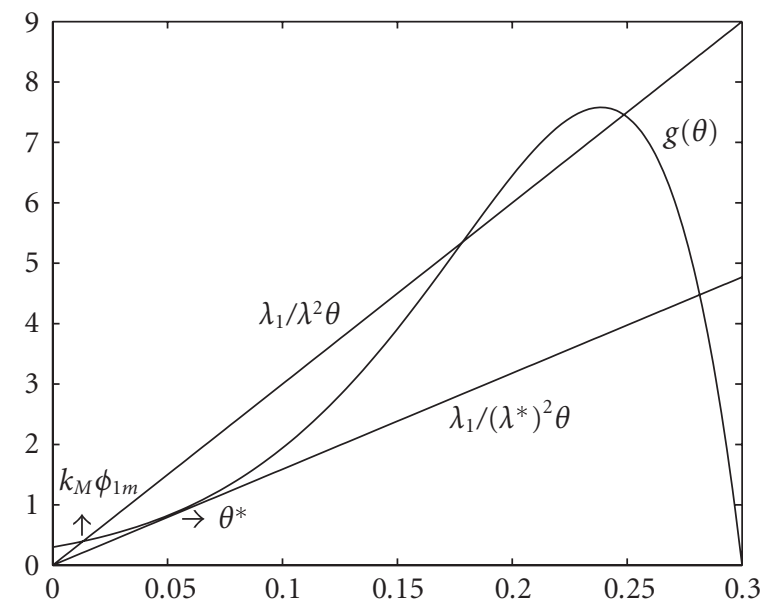

Figure 3.1. The values of $\theta^{*}$ and $k_{M}$, for $\beta=0.3$ and $\delta=25$.

Proof. Since $k_{0} \leq k_{M}$ and $\left(\lambda_{1} / \lambda^{2}\right) \phi_{1 m} u \leq g\left(\phi_{1 m} u\right)$ for $0 \leq u \leq k_{M}$, we have $k(t)$ increasing with equilibrium value $k_{M}$, that is, $k_{0} \leq k(t) \leq k_{M}$. Now,

$$
\begin{aligned}
P w & =k^{\prime}(t) \phi_{1}+\lambda_{1} k(t) \phi_{1}-\lambda^{2} g\left(k(t) \phi_{1}\right) \\
& =\frac{\phi_{1}}{\phi_{1 m}}\left\{\lambda^{2} g\left(k \phi_{1 m}\right)-\lambda_{1} k \phi_{1 m}\right\}+\lambda_{1} k \phi_{1}-\lambda^{2} g\left(k \phi_{1}\right) \\
& \leq\left(\lambda^{2} g\left(k \phi_{1 m}\right)-\lambda_{1} k \phi_{1 m}\right)-\left(\lambda^{2} g\left(k \phi_{1}\right)-\lambda_{1} k \phi_{1}\right) .
\end{aligned}
$$

Since $g(\theta)-\left(\lambda_{1} / \lambda^{2}\right) \theta$ is decreasing in $\left[0, \theta^{*}\right]$ for $\lambda \leq \lambda^{*}$, we have $P w \leq 0$ and the result is obtained.

Theorem 3.5. For $\lambda>\lambda^{*}$, let $\epsilon(\lambda)>1$ be such that $g\left(\theta^{*}\right)=\epsilon\left(\lambda_{1} / \lambda^{2}\right) \theta^{*}$, and let $k(t)$ be the solution of

$$
\begin{gathered}
k^{\prime}(t)=\frac{1}{\phi_{1 m}}\left\{\lambda^{2} g\left(k(t) \phi_{1 m}\right)-\epsilon \lambda_{1} k(t) \phi_{1 m}\right\}, \\
k(0)=k_{0},
\end{gathered}
$$

where $k_{0} \leq \theta^{*} / \phi_{1 m}$ is chosen such that $k_{0} \phi_{1}(\mathbf{x}) \leq r(\mathbf{x})$. Then the function $h(\theta)=g(\theta)-$ $\epsilon\left(\lambda_{1} / \lambda^{2}\right) \theta$ is decreasing in $\left[0, \theta^{*}\right]$ and $w(\mathbf{x}, t)=k(t) \phi_{1}(\mathbf{x})$ is a lower solution of $(2.1)$.

Proof. Since $\epsilon=\left(\lambda / \lambda^{*}\right)^{2}$, we have $h(\theta)=g(\theta)-\left(\lambda_{1} /\left(\lambda^{*}\right)^{2}\right) \theta$ and $h^{\prime}(\theta)=g^{\prime}(\theta)-\lambda_{1} /\left(\lambda^{*}\right)^{2} \leq$ $g^{\prime}\left(\theta^{*}\right)-\lambda_{1} /\left(\lambda^{*}\right)^{2}=0$. Using the same arguments as in the previous theorem, one can verify that $k(t)$ is increasing in $k_{0} \leq k(t) \leq \theta^{*} / \phi_{1 m}$. Since $g(\theta)-\epsilon\left(\lambda_{1} / \lambda^{2}\right) \theta$ is decreasing 
in $\left[0, \theta^{*}\right]$ and $\epsilon>1$ we have

$$
\begin{aligned}
P w & =k^{\prime}(t) \phi_{1}+\lambda_{1} k(t) \phi_{1}-\lambda^{2} g\left(k(t) \phi_{1}\right) \\
& =\frac{\phi_{1}}{\phi_{1 m}}\left(\lambda^{2} g\left(k \phi_{1 m}\right)-\epsilon \lambda_{1} k \phi_{1 m}\right)-\left(\lambda^{2} g\left(k \phi_{1}\right)-\lambda_{1} k \phi_{1}\right) \\
& \leq \lambda^{2} g\left(k \phi_{1 m}\right)-\epsilon \lambda_{1} k \phi_{1 m}-\left(\lambda^{2} g\left(k \phi_{1}\right)-\epsilon \lambda_{1} k \phi_{1}\right) \\
& =\lambda^{2}\left(\left[g\left(k \phi_{1 m}\right)-\epsilon \frac{\lambda_{1}}{\lambda^{2}} k \phi_{1 m}\right]-\left[g\left(k \phi_{1}\right)-\epsilon \frac{\lambda_{1}}{\lambda^{2}} k \phi_{1}\right]\right) \leq 0,
\end{aligned}
$$

which proves the result.

\section{Numerical results}

We consider the case where $g(\theta)$ is decreasing, and use Theorems 3.1 and 3.2 to obtain lower and upper solutions of (2.1). We present the bounds for different values of $\lambda, \beta, \delta$ and $0 \leq t \leq 0.2$, with initial condition $\theta(\mathbf{x}, 0)=\lambda^{2} \beta \psi(\mathbf{x})$, where $\psi(\mathbf{x})$ is defined in Theorem 3.2. Figures 4.1, 4.2, and 4.3 depict these bounds of the problem when $\Omega$ is the slab, unit sphere, and infinite cylinder, respectively, and $\beta=0.5, \delta=0.1$, and $\lambda=$ $0.5,1,2,5$. In order for the condition $k_{0}\left(\phi_{1} / \phi_{1 m}\right) \leq r(\mathbf{x})$ to be satisfied in Theorem 3.1, we take $k_{0}=\lambda^{2} \beta / 8, \lambda^{2} \beta / 18.9, \lambda^{2} \beta / 6.4$, for the slab, sphere, and infinite cylinder, respectively. From the figures one can see that the upper bounds are increasing in time, where

$$
h(t)=\lambda^{2} \beta+\frac{1}{\lambda^{2}} \frac{1}{1-\delta \beta}\left[1-e^{-\lambda^{2}(1-\delta \beta) t}\right]
$$

whereas the lower bounds are decreasing or increasing in time, depending on the geometry $k_{0}$ and $k_{m}$. For example, the lower solutions are decreasing in time in the slab geometry since $k_{0}>k_{m}$, while they are increasing with time for $\lambda=0.5,1,2$ and decreasing for $\lambda=5$ in the sphere. Table 4.1 shows $k_{0}$ and $k_{m}$, for $\beta=0.5, \delta=0.1$, and different values of $\lambda$. Also, the upper and lower solutions are close to each other and give good information about the exact solution $\theta$.

From Proposition 2.1 we have that if $\theta_{t}(\mathbf{x}, 0) \neq 0$, then the solution $\theta$ is either increasing or decreasing in time. We now take $\theta(\mathbf{x}, 0)=c \phi_{1}(\mathbf{x})$ and ask the following: for what values of $c$ the solution $\theta$ of (2.1) is increasing with respect to time? To answer the question, we substitute $k_{0}=c \phi_{1 m}$ in Theorem 3.1. Since $\theta(\mathbf{x}, 0)=w(\mathbf{x}, 0)$ and $w(\mathbf{x}, t)$ is increasing in time for $c<k_{m} / \phi_{1 m}$, then so is $\theta$.

Finally, we compare our bounds with the bounds obtained in [10]. Consider the PDE

$$
\begin{gathered}
\frac{\partial v}{\partial t}=\nabla^{2} v-\lambda^{2} c_{0} v+\mu_{0} \beta, \quad \mathbf{x} \in \Omega, \\
v(\mathbf{x}, t)=0, \quad \mathbf{x} \in \partial \Omega, \\
v(\mathbf{x}, 0)=r(\mathbf{x}) \geq 0,
\end{gathered}
$$

where $c_{0}=\max \left\{-\lambda^{2} g^{\prime}(v), 0 \leq v \leq \beta\right\}$. Let $\bar{v}(\mathbf{x}, t)$ be the solution of (4.2) with $\mu_{0}=c_{0}$, then $\bar{v}(\mathbf{x}, t)$ is an upper solution of (2.1). A lower solution $\underline{v}(\mathbf{x}, t)$ of $(2.1)$ is obtained by solving (4.2) with $\mu_{0}=\lambda^{2}$. For more details one can see [10, page 36]. For the slab 
Bounds for a reactive-diffuse system

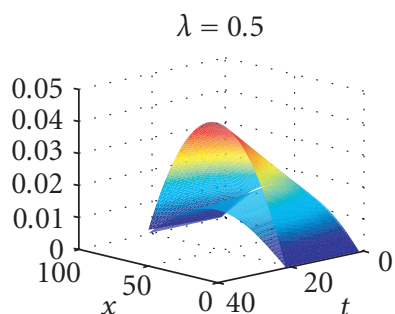

(a)

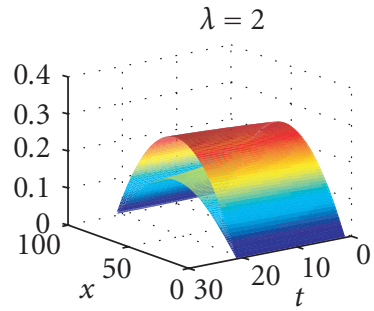

(c)

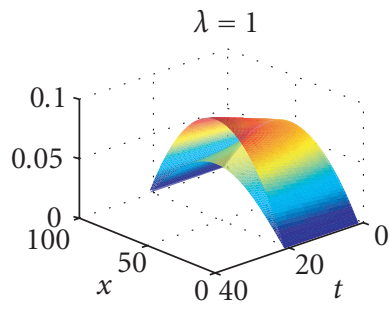

(b)

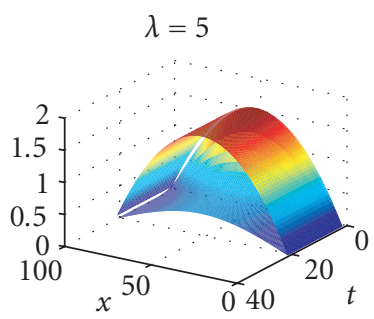

(d)

Figure 4.1. Upper and lower bounds for the slab geometry, when $\beta=0.5, \delta=0.1$, and $\lambda=0.5,1,2,5$.

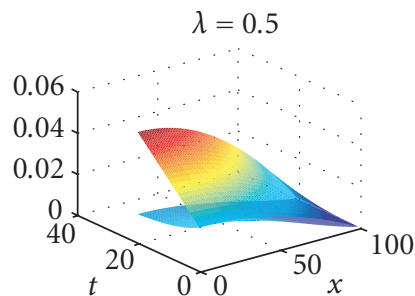

(a)

$$
\lambda=2
$$

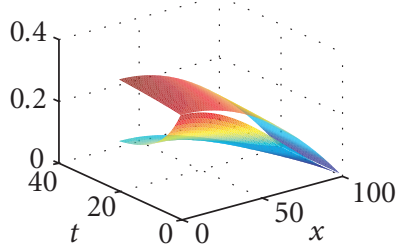

(c)

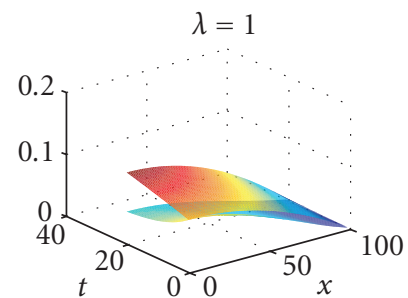

(b)

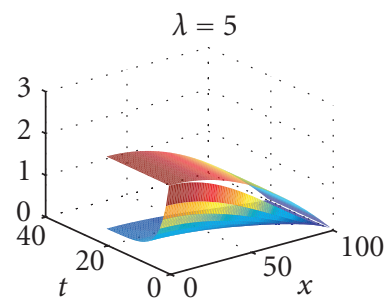

(d)

Figure 4.2. Upper and lower bounds for the spherical geometry, when $\beta=0.5, \delta=0.1$, and $\lambda=$ $0.5,1,2,5$. 
$\lambda=0.5$

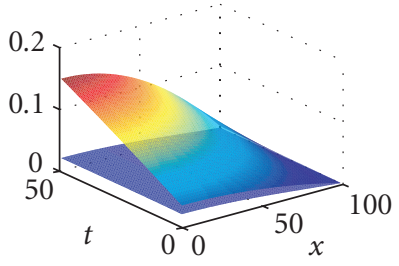

(a)

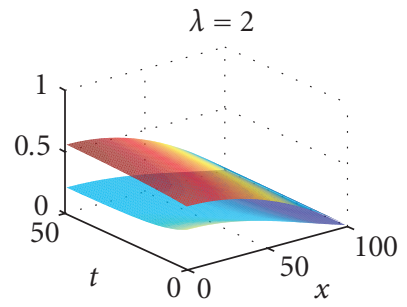

(c)

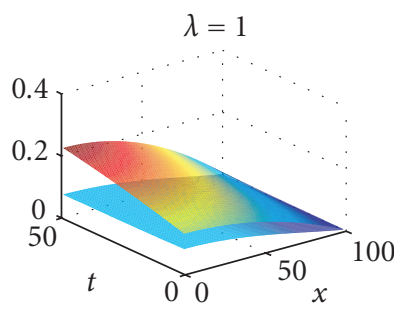

(b)

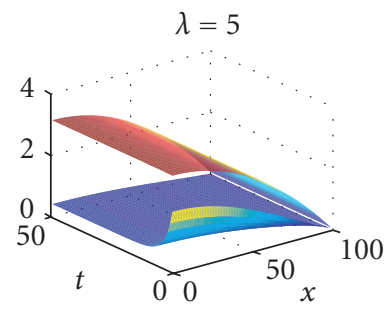

(d)

Figure 4.3. Upper and lower bounds for the cylindrical geometry, when $\beta=0.5, \delta=0.1$, and $\lambda=$ $0.5,1,2,5$.

Table 4.1. The values of $k_{0}$ and $k_{m}$, for $\beta=0.5$ and $\delta=0.1$, in the three geometries.

\begin{tabular}{l|c|cccc}
\hline & $\lambda$ & 0.5 & 1.0 & 2.0 & 5.0 \\
\hline \multirow{2}{*}{ Slab } & $k_{0}$ & 0.015625 & 0.062500 & 0.250000 & 1.562500 \\
& $k_{m}$ & 0.012367 & 0.046185 & 0.145507 & 0.361155 \\
\hline \multirow{2}{*}{ Sphere } & $k_{0}$ & 0.006614 & 0.026455 & 0.105820 & 0.661380 \\
& $k_{m}$ & 0.012367 & 0.046185 & 0.145507 & 0.361155 \\
\hline \multirow{2}{*}{ Cylinder } & $k_{0}$ & 0.019531 & 0.078125 & 0.312500 & 1.953100 \\
& $k_{m}$ & 0.020759 & 0.074147 & 0.206504 & 0.408257 \\
\hline
\end{tabular}

geometry we have

$$
\begin{gathered}
\bar{v}(x, t)=\frac{2 \beta}{\pi} \sum_{n=1}^{\infty} \frac{1-(-1)^{n}}{n}\left(\frac{\lambda^{2}}{n^{2} \pi^{2}} e^{-r_{n} t}+\frac{\mu_{0}}{r_{n}}\left(1-e^{-r_{n} t}\right)\right) \sin (n \pi x), \\
\underline{v}(x, t)=\frac{2 \beta \lambda^{2}}{\pi} \sum_{n=1}^{\infty} \frac{1-(-1)^{n}}{n}\left(\frac{1}{n^{2} \pi^{2}} e^{-r_{n} t}+\frac{1}{r_{n}}\left(1-e^{-r_{n} t}\right)\right) \sin (n \pi x),
\end{gathered}
$$

where $r_{n}=n^{2} \pi^{2}+\lambda^{2} \mu_{0}$ and $\mu_{0}=1.033895$.

Figures 4.4 and 4.5 on the left show the upper solutions $u$ and $\bar{v}$ and on the right the difference between them. For $\lambda=0.1$ and 0.5 we have $\bar{v}-u \geq 0$, that is, the upper 


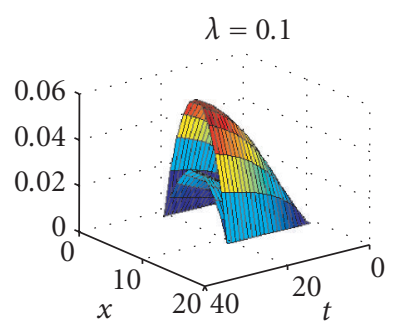

(a)

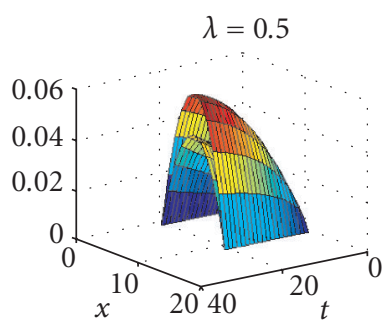

(c)

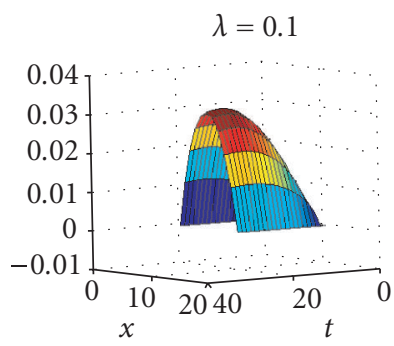

(b)

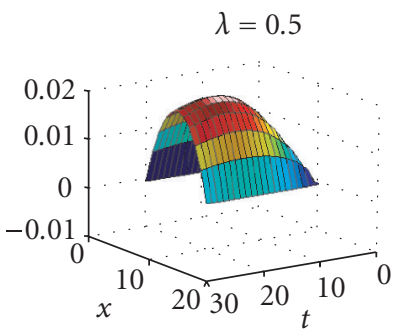

(d)

Figure 4.4. The upper solutions $u(\mathbf{x}, t)$ and $\bar{v}(\mathbf{x}, t)$ and the difference $\bar{v}(\mathbf{x}, t)-u(\mathbf{x}, t)$ for the slab geometry, when $\beta=0.5, \delta=0.1$, and $\lambda=0.1,0.5$.

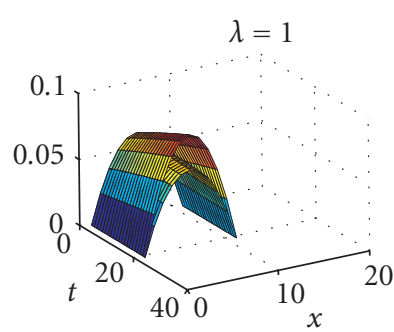

(a)

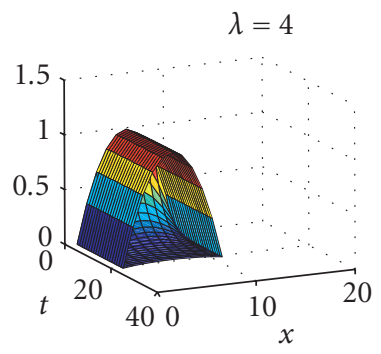

(c)

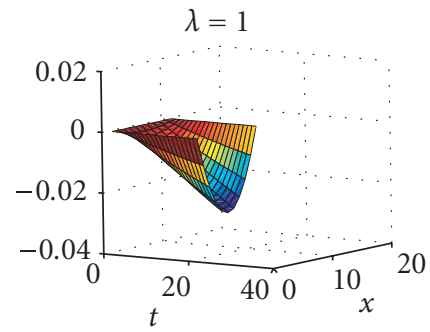

(b)

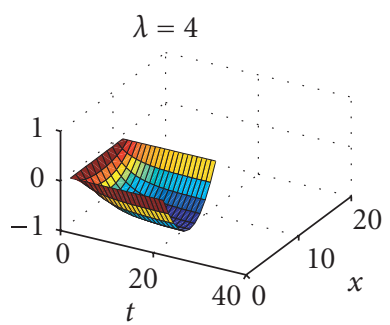

(d)

Figure 4.5. The upper solutions $u(\mathbf{x}, t)$ and $\bar{v}(\mathbf{x}, t)$ and the difference $\bar{v}(\mathbf{x}, t)-u(\mathbf{x}, t)$ for the slab geometry, when $\beta=0.5, \delta=0.1$, and $\lambda=1,4$. 


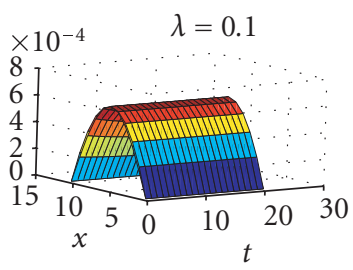

(a)

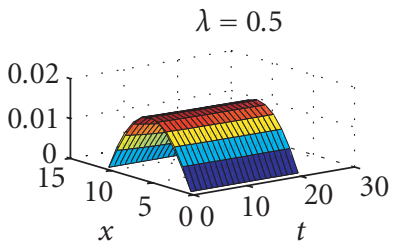

(c)

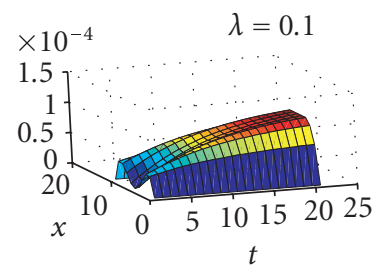

(b)

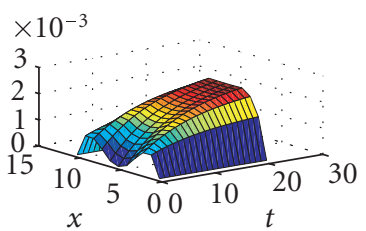

(d)

Figure 4.6. The lower solutions $w(\mathbf{x}, t)$ and $\underline{v}(\mathbf{x}, t)$ and the difference $\underline{v}(\mathbf{x}, t)-w(\mathbf{x}, t)$ for the slab geometry, when $\beta=0.5, \delta=0.1$, and $\lambda=0.1,0.5$.

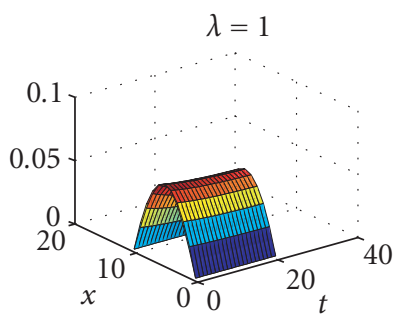

(a)

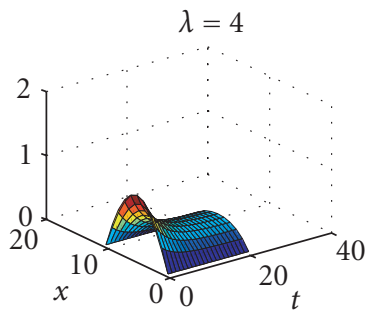

(c)

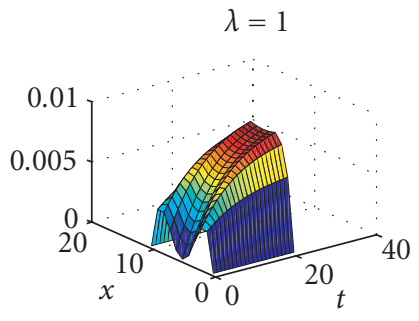

(b)

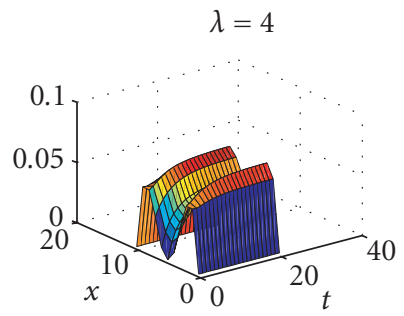

(d)

Figure 4.7. The lower solutions $w(\mathbf{x}, t)$ and $\underline{v}(\mathbf{x}, t)$ and the difference $\underline{v}(\mathbf{x}, t)-w(\mathbf{x}, t)$ for the slab geometry, when $\beta=0.5, \delta=0.1$, and $\lambda=1,4$.

solution $u$ is better than $\bar{v}$. Whereas for $\lambda=1$ and 4 we have $u \geq \bar{v}$, and the upper solution $\bar{v}$ is better than $u$. Figures 4.6 and 4.7 on the left show the lower solutions $w$ and $\underline{v}$ and on 
the right the difference between them. One can see that the two bounds are close to each other and for all values of $\lambda$ we have $\underline{v} \geq w$, that is, the lower solution $\underline{v}$ is better than $w$.

\section{Concluding remarks}

We have used comparison arguments to study a nonlinear parabolic equation arising from the theory of catalyst pellets reaction. For $\delta \beta \leq 1$, a lower solution of the form $w(\mathbf{x}, t)=k(t)\left(\phi_{1} / \phi_{1 m}\right)$ is obtained, where $\phi_{1}$ is the first normalized eigenfunction of the associated Laplacian operator, $\phi_{1 m}$ is the maximum of $\phi_{1}$ in $\Omega$, and $k(t)$ is the solution of an IVP. Depending on the initial condition $k(0)$, the function $k(t)$ might be decreasing or increasing. An upper solution of the form $u(\mathbf{x}, t)=h(t) \psi(\mathbf{x})$ is obtained by solving a second-order linear IVP for $h(t)$ and a linear PDE for $\psi$, where $h(t)$ is increasing in time. The lower solution is used to give a sufficient condition for the solution $\theta$ to be increasing in time for certain initial condition. For the case where $\delta>4+4 / \beta$, we have constructed a lower solution $w(\mathbf{x}, t)=k(t) \phi_{1}(\mathbf{x})$, where $k(t)$ is increasing and depends on the value of $\lambda^{*}$. We present the upper and lower solutions for certain parameters in the three geometries numerically. These upper and lower solutions are compared with the ones obtained by Pao [10] for the slab geometry.

\section{References}

[1] M. Al-Refai, Existence, uniqueness and bounds for a problem in combustion theory, Journal of Computational and Applied Mathematics 167 (2004), no. 2, 255-269.

[2] __ Bounds and critical parameters for a combustion problem, Journal of Computational and Applied Mathematics 188 (2006), no. 1, 33-43.

[3] R. Aris, The Mathematical Theory of Diffusion and Reaction in Permeable Catalysts, Vol. I, Clarendon Press, Oxford, 1975.

[4] J. Bebernes and D. Eberly, Mathematical Problems from Combustion Theory, Applied Mathematical Sciences, vol. 83, Springer, New York, 1989.

[5] D. W. Drott and R. Aris, Communications on the theory of diffusion and reaction-I: a complete parametric study of the first-order, irreversible exothermic reaction in a flat slap of catalyst, Chemical Engineering Science 24 (1969), no. 3, 541-551.

[6] A. K. Kapila and B. J. Matkowsky, Reactive-diffuse systems with Arrhenius kinetics: multiple solutions, ignition and extinction, SIAM Journal on Applied Mathematics 36 (1979), no. 2, 373-389.

[7] __ Reactive-diffusive system with Arrhenius kinetics: the Robin problem, SIAM Journal on Applied Mathematics 39 (1980), no. 3, 391-401.

[8] A. K. Kapila, B. J. Matkowsky, and J. Vega, Reactive-diffusive system with Arrhenius kinetics: peculiarities of the spherical geometry, SIAM Journal on Applied Mathematics 38 (1980), no. 3, 382-401.

[9] M. L. Michelsen and J. Villadsen, Diffusion and reaction on spherical catalyst pellets: steady state and local stability analysis, Chemical Engineering Science 27 (1972), no. 4, 751-762.

[10] C. V. Pao, Nonlinear Parabolic and Elliptic Equations, Plenum Press, New York, 1992.

[11] M. H. Protter and H. F. Weinberger, Maximum Principles in Differential Equations, Springer, New York, 1984. 
[12] D. H. Sattinger, Monotone methods in nonlinear elliptic and parabolic boundary value problems, Indiana University Mathematics Journal 21 (1972), no. 11, 979-1000.

Mohammed Al-Refai: Department of Mathematics and Statistics, Jordan University of Science and Technology, P.O. Box 3030, Irbid 22110, Jordan

E-mail address: m_alrefai@yahoo.com

Qutaibeh Katatbeh: Department of Mathematics and Statistics, Jordan University of Science and Technology, P.O. Box 3030, Irbid 22110, Jordan

E-mail address: quaibeh@yahoo.com 


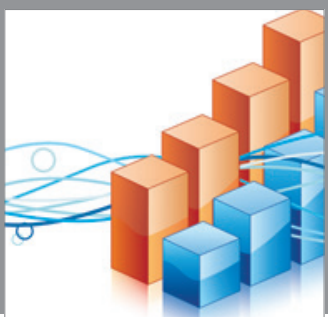

Advances in

Operations Research

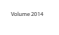

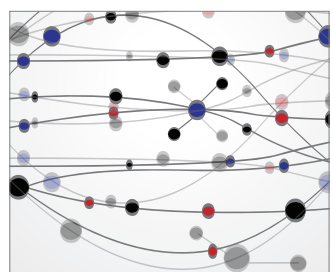

\section{The Scientific} World Journal
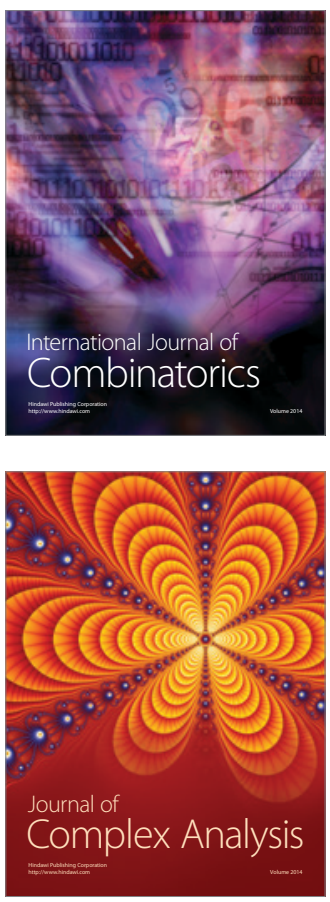

International Journal of

Mathematics and

Mathematical

Sciences
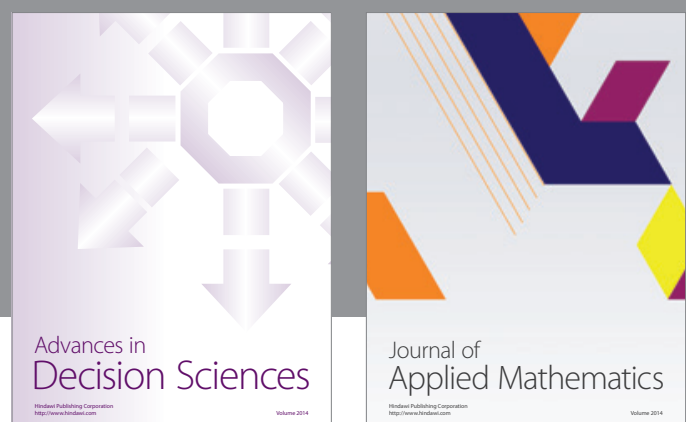

Journal of

Applied Mathematics
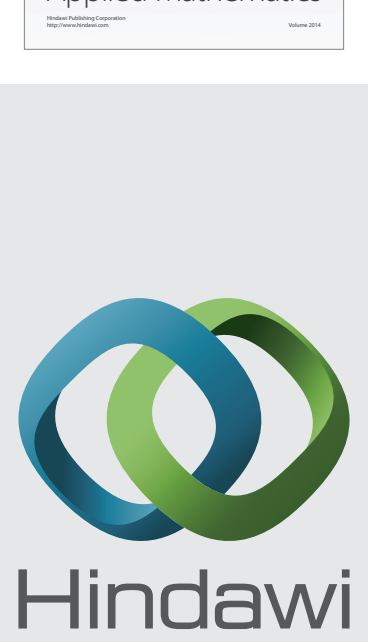

Submit your manuscripts at http://www.hindawi.com
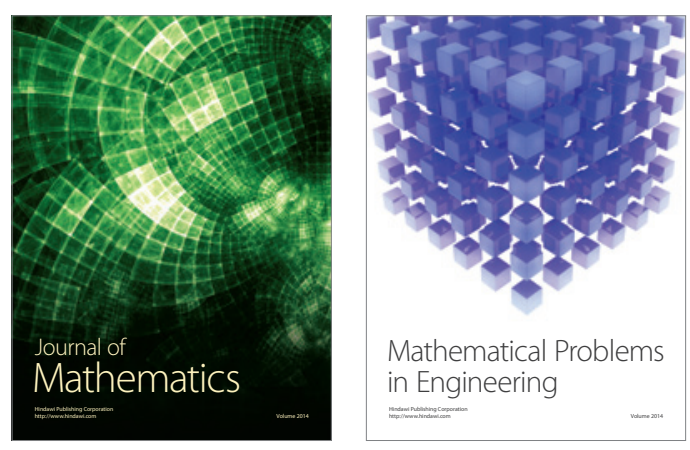

Mathematical Problems in Engineering
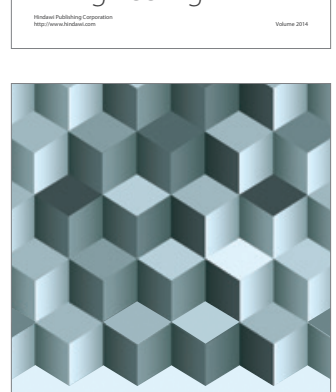

Journal of

Function Spaces
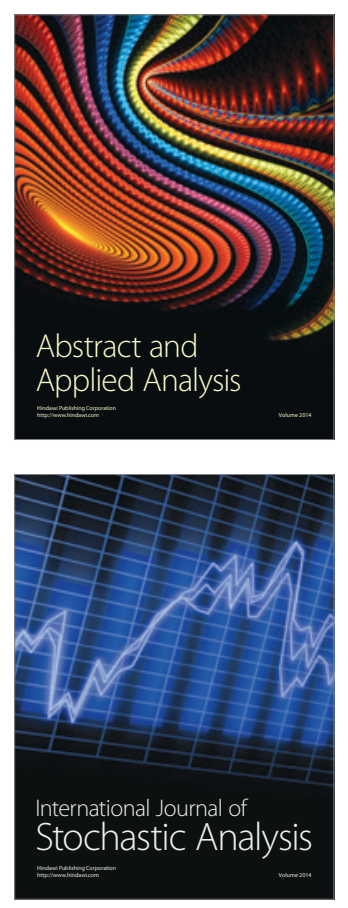

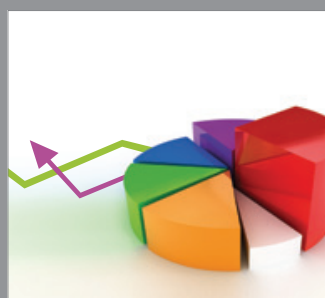

ournal of

Probability and Statistics

Promensencen
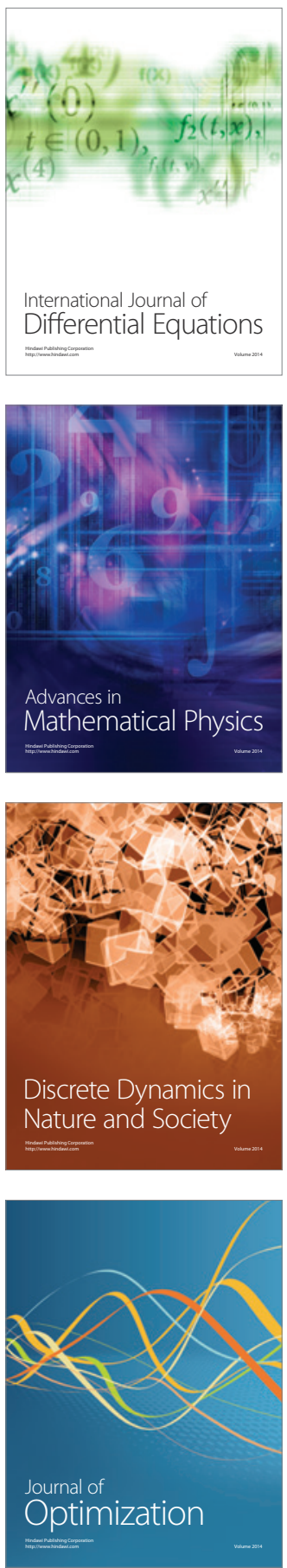